\title{
Cyst of traumatic origin
}

\begin{abstract}
The traumatic bone cyst is an intraosseous cavity related to the jawbones and is devoid of epithelial tissue and has been reported in the literature under a variety of names. It is a rare pathology affecting the mandible more than the maxilla. Its onset occurs mainly during the first two decades of life, irrespective of sex with an excellent prognosis.
\end{abstract}

Keywords: trauma, intraosseous, cyst, mandible
Volume 8 Issue 3 - 2018

\author{
Sreelakshmi N Nair,' Raghavendra Kini,' \\ Prasanna Kumar Rao, ${ }^{2}$ Devika N Shetty, \\ Gowri Bhandarkar,' Roopashri R Kashyap' \\ 'Department of Oral Medicine and Radiology,AJ Institute of \\ Dental Sciences, India \\ ${ }^{2}$ Department of Oral and Maxillofacial Surgery, AJ Institute of \\ Dental Sciences, India
}

Correspondence: Sreelakshmi N Nair, Department of Oral Medicine and Radiology, AJ Institute of Dental Sciences, India, Tel +917829030464, Email sreelakshminnair@gmail.com

Received: November 28, 2017 | Published: May II, 2018

\section{Introduction}

Traumatic bone cyst (TBC), is a rare and infrequent intraosseous non neoplastic cyst. It is not considered as a true cyst because it lacks a well defined epithelial lining. It was first described by Virchow in 1876. It was Lucas and Blum who first used the term in $1929 .{ }^{1}$ The traumatic bone cyst first described many decades ago, has been appellated with a myriad of names including: simple bone cyst, solitary bone cyst, hemorrhagic bone cyst, extravasation cyst, idiopathic bone cyst, and primary bone cyst. However, the term TBC is more widely used in the literature. ${ }^{2}$ This variation in terminology reflects the unclear etiology and pathogenesis of traumatic bone cysts. Among the different etiological possibilities, trauma has been considered most common. They are asymptomatic and are commonly discovered on accidental radiographic findings. We hereby report a similar asymptomatic case found on accidental radiographic finding.

\section{Case report}

A 21 year old medically fit male patient visited our dental department with a chief complaint of a decayed tooth in relation to the lower left back tooth region since 6 months. Patient gave a history of pain in relation to the same on biting on hard food which relieved at rest. Pain was intermittent, pricking type, non radiating in nature. Patient gave a history of being hospitalized a year back following a sports injury where he hit his left side of the face on the ground, for which symptomatic treatment was given. His family history was non contributary. There was no abnormality detected on extraoral examination. On intraoral examination, there was a grossly decayed tooth in relation to the lower left back tooth region involving enamel and dentin and appeared brownish black in colour. No other soft tissue abnormalities were detected or any vestibular space obliteration in relation to the same region. Based on and clinical presentation, a provisional diagnosis of deep dentinal caries in relation to lower left back tooth region was given. Intra oral periapical radiographic (IOPA) examination revealed loss of crown structure and radiolucency involving enamel, dentin and approximating pulp with periapical widening of periodontal ligament space in relation to lower left back tooth region. A well defined radiolucency with a well defined sclerotic border was seen in relation to the root apices of lower left back tooth region extending from 35 to 37 region which scalloped in between the roots superiorly and extended till the inferior border of mandible inferiorly. An Orthopantamograph (OPG) was taken to confirm the extent of the lesion and it revealed the same. On noticing the incidental radiographic finding, an Electric pulp vitality test was done to confirm the vitality of the teeth involved in the region. It was found that there was normal pulpal response in relation to 36 and delayed response in relation to 35 and 37 showing that these teeth were vital.

Based on the history of trauma, radiological findings and findings of pulp testing, a diagnosis of traumatic bone cyst in relation to 35 , 36,37 region was given and apical periodontitis in relation to 37 was given. A fine needle aspiration and incisional biopsy was performed which microscopically revealed bone tissue and extravasated RBCs. Lining epithelium was not evident. All these features were suggestive of traumatic bone cyst. So taking into account the clinical, radiological and histopathological examination, a final diagnosis of traumatic bone cyst in relation to $35,36,37$ region was given.

\section{Discussion}

Traumatic bone cyst, a pathological cavity of complex etiology was first discovered by Virchow in the year 1876 and described by Lucas in the year 1929.It was classified by WHO in 2005 as a non-neoplastic osseous lesion. It shows no epithelial lining, which sets apart this lesion from the true cysts. ${ }^{3}$ Barnes enlisted innumerable terms for traumatic bone cyst which was familiarized in literature as traumatic cyst, traumatic haemorhagic cyst, progressive bone cavity, solitary bone cavity, solitary bone cyst, simple bone cyst, idiopathic bone cavity, haemorhagic bone cyst, extravasation cysts, haemorhagic cyst, haemorhagic extravasation, solitary haemorhagic cyst. ${ }^{4}$ This array of countless names is as a consequence of lack of clear understanding of its etiology and pathogenesis. The varied etiological factors which have been put forth are tumor elicited bone degeneration, calcium metabolism alterations, local alteration in bone growth, venous 
obstruction, osteolysis increase, intramedullary bleeding, local ischaemia, mild infectious process, bone marrow infection, lymphatic drainage obstruction from sinusoidal veins leading to trabecular bone resorption. ${ }^{5}$ Out of this, trauma is the most accepted etiological factor responsible for the cyst evolution. It is found that a history of injury of the affected part of the jaw is elicited in most cases which may have occurred long back before the discovery of the lesion. In our case too, patient gave a history of sports injury where he hit his left side of the face against the floor. ${ }^{6}$ The most widely accepted theory suggests that bleeding within the bone caused by trauma causes organization of a blood clot and healing, the clot liquefactively necrotises resorbs. The surrounding bone is destroyed by enzymatic activity. Due to the increased pressure of its content bone cavity enlarges resulting in cyst formation. ${ }^{7}$ The most affected population is the patients between 10 and 20 years of age with a higher prevalence among men than women. In our case too, a 21 year old male patient was affected. It is more commonly found in the posterior portion of the mandible which was true in our case too where the lower left back tooth region was affected. ${ }^{8}$

Even in the cases where the lesion is associated with roots of teeth, there is no increased mobility of the teeth or changes in their color. The present case too had normal appearing teeth associated with the lesion. Pulp vitality is not likely to be altered. However, due to traumatic force, cystic expansion may increase the root pressure resulting in a temporary reduction in the pulpal response to the electric test which holds good in our case too. ${ }^{9-15}$ Clinically, there are no soft tissue changes, pain or paresthesia. In the early phases, there is usually no bone expansion while in the later phase; there can be bone expansion. In our case there was no bone expansion. ${ }^{12}$ Periapical radiographs are ideal for early radiographic assessment but these radiographs fail to show the whole extent of the lesion. Panoramic radiography, computed tomography and magnetic resonance are more precise imaging modalities in the diagnosis of these cysts. In our case the cyst was an incidental finding in an IOPA the extent of which was found later by an OPG. ${ }^{8}$ Radiographically, these lesions appear as a well defined radiolucent defect with diameter in the range of range 1 to $10 \mathrm{~cm}$ as seen in our case. ${ }^{10}$ The cysts usually presents as scalloping pattern between the roots of the involved teeth which was seen in our case too where the cyst scalloped between the roots of 36 and $37 .{ }^{11}$ The structure of the mandible canal may in some cases be preserved and in other cases not. There is no resorption of the teeth roots. The lamina dura may or may not be involved. ${ }^{4}$ In our case, the lesion involved the mandibular canal, with loss of lamina dura but no root resorption. Histopathological examination reveals a thin band of vascular fibrous connective tissue lacking an epithelial lining. In our case also histopathological examination revealed bone tissue and extravasated RBCs without lining epithelium. ${ }^{13}$ Treatment is surgical operation which consists of evacuation of the content, cochleation of the cavity in order to stimulate bleeding in the cavity. The wound is then sutured. This is followed by the formation/creation and organisation of a clot, and healing by the formation of new bone. ${ }^{14}$ In our case also treatment consisted of bone curettage under local anesthesia and surgical closure of the wound. The patient will be monitored clinically and radiographically every 6 months to follow the progression of healing. Healing occurs about 6 to 24 months after bleeding and closure of the area. Prognosis is usually excellent with rare chance of recurrence ${ }^{15}$ ( Figure 1-4).

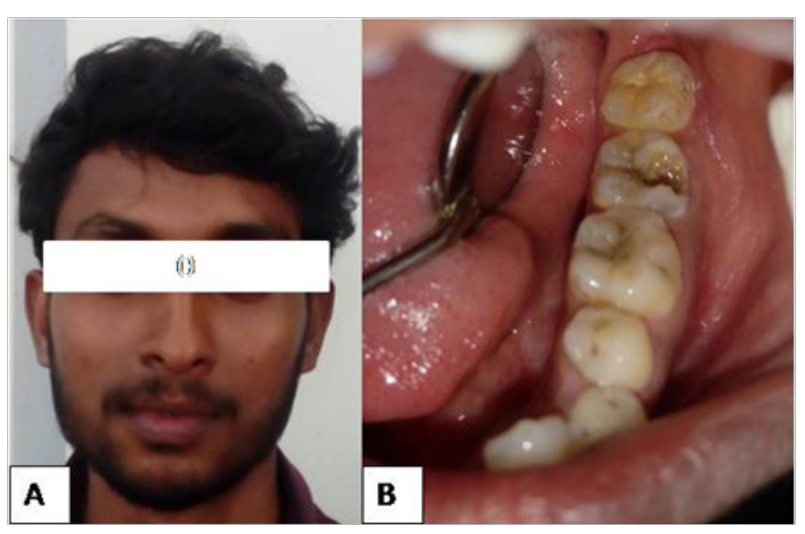

Figure 1 A- showing the profile of the patient. B- showing the area of interest showing a decayed tooth and no cortical involvement.

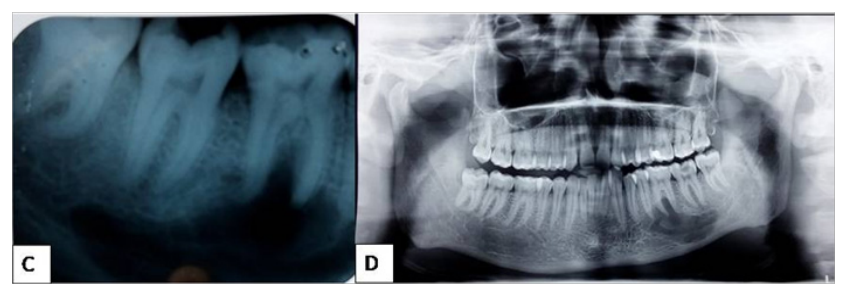

Figure 2 C- showing the extent of a well defined radiolucency in the IOPA of mandibular left back tooth region. D- showing the same on an OPG.

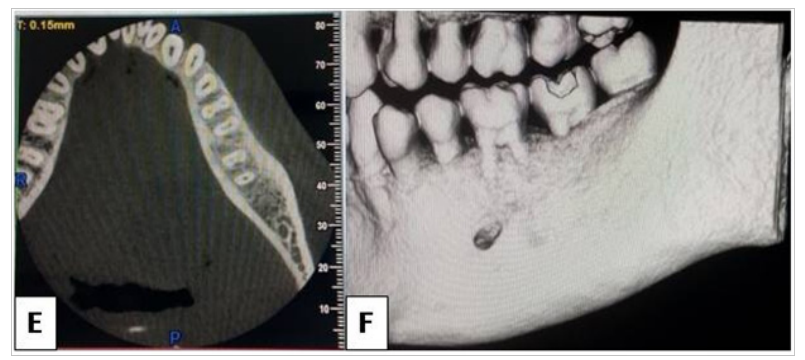

Figure 3 E- showing axial section of a CBCT image showing no cortical expansion. F- 3D section of the same showing horizontal bone loss and no cortcal expansion.

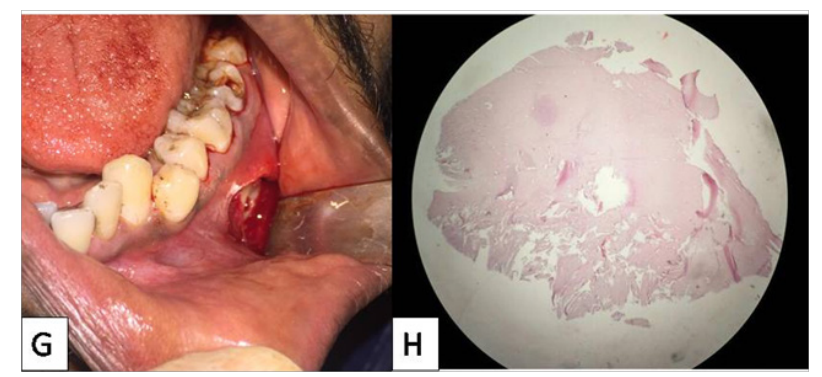

Figure 4 G- showing incisional biopsy of the lesion. H- histopathological picture showing revealed bone tissue without lining epithelium was not evident.

\section{Conclusion}

Traumatic bone cyst has an indefinite etiology and is considered as a pseudocyst because of the lack of an epithelial lining. The final 
diagnosis should be established considering clinical, radiographic, and histological findings. Even though the recurrence rate is low, radiographic follow-up for one or two years after surgical treatment is necessary for the confirmation of a possible cure or recurrence.

\section{Acknowledgements}

None.

\section{Conflict of interest}

The author declares there is no conflict of interest.

\section{References}

1. Agrawal SG, Nayyar Abhishek S, Panda Arun, et al. Traumatic Bone Cyst: Is Trauma Always the Cause or, Symptoms Always Lacking? A Case Report of an Unusual Case. International Journal of Clinical Case Reports. 2015;5(30):1-4.

2. Beasley JD. Traumatic cyst of the jaws: report of 30 cases. J Am Dent Assoc. 1976;92(1):145-52.

3. Velasco I, Cifuentes J, Lobos N, et al. The unusual evolution of a simple bone cyst in the mandible: a case report. J Clin Exp Dent. 2012;4(2):1325.

4. Sandev S, Sokler K, Grgurevi J. Traumatic Bone Cysts. Acta Stomat Croat. 2001;35(3):417-20.

5. Wong-Romo G. Solitary mandibular bone cyst. Revista Odontológica Mexicana. 2016;20(2):112-9.

6. Salem AS, Abdelfadil E, Mourad S, et al. The Traumatic Etiology Hypothesis of Traumatic Bone Cyst: Overview and Report of a Case. Int Journ of Clin Case Report. 2015;5(30):1-4.

7. Kumar S, S Padmashree, Jayalekshmy Rema. Traumatic bone cyst of idiopathic origin? a report of two cases. Ethiop J Health Sci. 2014;24(2):183-7.

8. Dias SL, Silva LF, Sande Vieira TSL, et al. Simple bone cyst: a case report and review of the literature. J Health Sci Inst. 2012;30(3):295-8.

9. Lago CA, Cauás M, Pereira AMP, et al. Cisto ósseo traumáticoem mandíbula: relato de caso. Rev Cir Traumatol Buco-Maxilo-Fac. 2006;6:17-22.

10. Ferreira Júnior FO, Damante JH, Lauris JR. Simple bone cyst versus odontogenic keratocyst: differential diagnosis by digitized panoramic radiography. Dentomaxillofac Radiol. 2004;33(6):373-8.

11. Suei Y, Taguchi A, Kurabayashi T, et al. Simple bone cyst: investigation on the presence of gas in the cavity using computed tomography. Oral Surg Oral Med Oral Pathol Oral Radiol Endod. 1998;86(5):592-4.

12. Forssell K, Forssell H, Happonen RP, Neva M. Simple bone cyst. Review of the literature and analysis of 23 cases. Int J Oral Maxillofac Surg. 1988;17(1):21-4.

13. Strabbing EM, Gortzak RA, Vinke JG, et al. An atypical presentation of a solitary bone cyst of the mandibular ramus: A case report. Journal of Cranio Maxillo Fac Surg. 2011;39:145-7.

14. Suei Y, Taguchi A, Tanimoto K. Simple bone cyst of the jaws: evaluation of treatment outcome by review of 132 cases. J Oral Maxillofac Surg. 2007;65(5):918-23.

15. Tong AC, Ng Io, Yan BS. Variations in clinical presentations of simple bone cyst: report of cases. J Oral Maxillofac Surg. 2003;61:1487-91. 